\title{
Understanding the Challenges and Opportunities with Big Data Applications over "Smart Healthcare System"
}

\author{
D. Saidulu \\ Associate Professor-CSE \\ Guru Nanak Institutions Technical Campus, \\ Hyderabad-Telangana, India
}

\author{
R. Sasikala, PhD \\ Associate Professor \\ School of Computer \\ Science \& Engineering (SCOPE) \\ VIT University, \\ Vellore-632014, \\ Tamilnadu, India.
}

\begin{abstract}
Objective: Nowadays, Big Data technologies play a vital role in diverse areas like health domain, finance, and social networking, and so forth. This paper enlightens the readers, students and research scholars to be aware of challenges and opportunities with Big Data applications over "Smart Healthcare System" for refining optimized patient-centric services.
\end{abstract}

Methods/Analysis: Big Data applications over the smart health care system with challenges and opportunities which support providers to identify and recommend appropriate medication in advance using Sensor Technology, machine learning, and Big Data techniques.

Findings: This paper introduces appropriate medications, augmenting in research by understanding the Big Data Challenges and Opportunities, which deal with patient data capture, store, search, sharing, transfer, analysis, and visualization.

Applications/Improvements: Big Data Analytics on Smart Health Care systems with sensor technology, which improves diagnosis, therapy, and cares at an advanced level, saves time and reduces the cost.

\section{Keywords}

Smart Healthcare System, Machine learning, Big Data, Sensor Technology, Big Data Analytics.

\section{INTRODUCTION}

conventionally when the healthcare domain traditionally produces massive volume of data constrained by self-possessed data capture, store, search, sharing, transfer, analysis, and patient care while most data is stored in the hardcopy format, the current trend is drastically improved towards digitization of this immense volume of data driven by required necessities and the possible to increase the eminence of healthcare provision, for the moment, reducing the costs and promise of supporting a wide range of medical and healthcare functions, including among other's clinical decision support, disease surveillance, and population health management.

A research study was made by IBM, which says that IBM [2] estimates 2.5 quintillion bytes ( 2.5 exabytes) of data are created every day from a variety of sources. Futuristic analysis by CISCO says that the projected increase of global Internet traffic in the middle of, 2015 and 2016 alone are more than 330 exabytes, which is almost equal to the total amount of global IP traffic generated in 2011 (369 exabytes)" which is a unrepeatable increase growth of data (Figure 1).

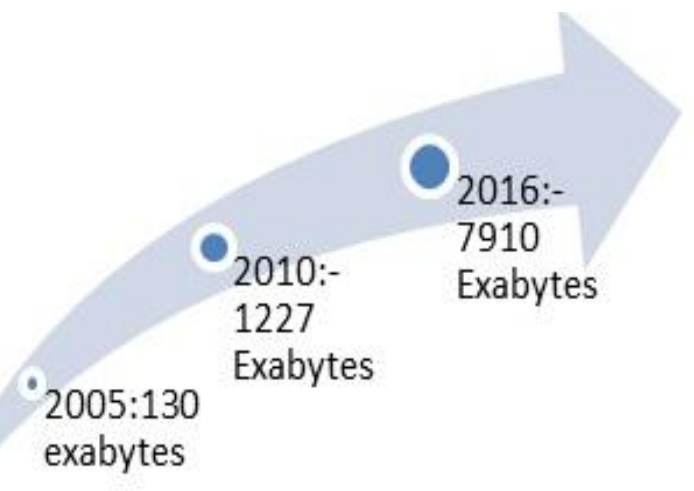

Figure 1 - Growth of data

Generated huge volume of health data hard to manage with outdated software and hardware; Big data in healthcare is incredible not only because of its volume but also due to the variety of data types and the promptness at which it must be managed [1]. It includes clinical data from clinical decision support systems patient data in electronic patient records (EPRs); machine generated/sensor data, such as from monitoring vital signs; likely for big data analytics in healthcare to lead to improved results survives via several consequences, The principal unbiased of Big Data Analytics in Smart Health Care System is to Costs diminish; advance diagnosis, analysis, and precaution.

\subsection{Big Data is typically highlighted by 6} V's

1. Volume: Storage of excessive data.

2. Velocity: Rate at which data is flowing. Information is flowing in at extraordinary speed.

3. Variety: grouping of data formats(text, images, audio, video, etc,.)

4. Veracity: uncertainty of data

5. Validity: correct data and accurate for the intended use.

6. Volatility: in what way data valid and how long should it be stored.

\subsection{Levels of Big Data Analytics}

Big Data analysis can do on four levels shown in (Figure 2)

1. Educate: it describes with data gathering and concern clarifications

2. Explore or discover: classify the relevant data from data source and support the concern needs and challenges. 
3. Engage: use statistical modelling or any analytical technique to make some insights out of Big Data

4. Implementation or Execute: Deploy the analytics for more Big Data initiatives

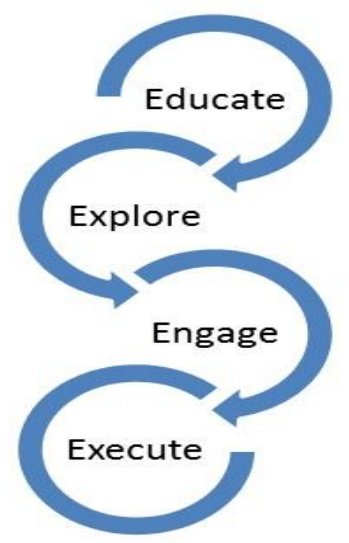

Figure 2.Levels of Analytics

This paper describes with the study of the Challenges and Opportunities with Big Data applications over "Smart Healthcare System." Primarily, we cover the comparative knowledge of Data Science, Big Data, and Data Analytics, Secondly, challenges. Privacy concerns in smart healthcare discovered. Thirdly, opportunities identified with big data applications. Fourthly discussed Primitive Mechanism of Smart Healthcare System, Fifthly discussed Big Data analytics and goals of Smart Healthcare System. Lastly, the conclusions presented.

\section{LITERATURE SURVEY}

Liang Y, Kelemen A (2016),"Big Data Science and Its Applications in Health and Medical Research: Challenges and Opportunities" [12]. In this literature, it explores the various levels of human life: individuals to the community, and industrial to university to government and also comes with many challenges in all fields, especially the biomedical and health science fields.

Devika Das 2016, "How To Improve Health Care Systems With IoT and Big Data"[11] in this literature we have been studying the application of machine learning techniques which helps clinicians and doctors make an accurate diagnosis; and also refers to historical medical data related to clinical assessments, lab values.

Raghupathi W, Raghupathi V(2014)[1].” Big data analytics in healthcare: promise and potential", The main objective of this article is to describe the promise and potential of big data analytics in healthcare. Which are evolving into a promising field for providing insight from enormous data sets and, improving outcomes while reducing costs?

\section{COMPARATIVE KNOWLEDGE OF DATA SCIENCE, BIG DATA, AND DATA ANALYTICS}

Before getting into Big Data analytics, we will clearly understand what is data science, Big Data, and Big Data Analytics as is seen in Table 1.
Table 1. Comparative Study on Data Science, Big Data, and Data Analytics

\begin{tabular}{|l|l|l|l|}
\hline & Data science & Big Data & $\begin{array}{l}\text { Data } \\
\text { Analytics }\end{array}$ \\
\hline $\begin{array}{l}\text { What is } \\
\text { what }\end{array}$ & $\begin{array}{l}\text { Processing to } \\
\text { extract } \\
\text { knowledge or } \\
\text { insights from } \\
\text { datar } \\
\text { Simply related } \\
\text { to data } \\
\text { cleansing and } \\
\text { analysis }\end{array}$ & $\begin{array}{l}\text { Extremely large } \\
\text { data sets that } \\
\text { analyzed be } \\
\text { computationally } \\
\text { to reveal } \\
\text { patterns and }\end{array}$ & $\begin{array}{l}\text { Reveal } \\
\text { patterns and } \\
\text { decision- } \\
\text { making in } \\
\text { business } \\
\text { moves at the } \\
\text { right } \\
\text { timeline. }\end{array}$ \\
\hline Usage & $\begin{array}{l}\text { Web search, } \\
\text { advertisements }\end{array}$ & $\begin{array}{l}\text { Mobile data, } \\
\text { medical data }\end{array}$ & $\begin{array}{l}\text { Healthcare, } \\
\text { Government, }\end{array}$ \\
\hline $\begin{array}{l}\text { Skills } \\
\text { required }\end{array}$ & $\begin{array}{l}\text { Working with } \\
\text { unstructured } \\
\text { data, in-depth } \\
\text { knowledge in } \\
\text { SAS, Python, } \\
\text { and Hadoop }\end{array}$ & $\begin{array}{l}\text { Creativity and } \\
\text { business skills }\end{array}$ & $\begin{array}{l}\text { Mathematics, } \\
\text { machine } \\
\text { learning } \\
\text { skills and } \\
\text { data } \\
\text { visualisation } \\
\text { skills }\end{array}$ \\
\hline
\end{tabular}

\section{BIG DATA: CHALLENGES, PRIVACY CONCERNS}

Perhaps healthcare is one of the most data-intensive organizations the challenge is not just in data capture, store, search, sharing, transfer, analysis, and visualization but also in making this data usable, above challenges can view in three dimensions i.e. Data, Process, and Management levels [2]

Data Challenges consist of Volume, Variety, Velocity, Veracity, Data Discovery, Data Assumptions, Data comprehensiveness and Data Scalability.

\section{Data challenges $\left[\mathbf{4 V}^{\mathrm{s}}, \mathbf{4} \mathrm{D}^{\mathrm{s}}\right]$}

1. Volume: It describes with size of Big Data

2. Variety: It handles multiple data type formats etc..

3. Velocity: It reacts with information overflow within a period.

4. Veracity: data reliability, data quality, data availability: it handles uncertainty, missing values, etc

5. Data discovery: it finds good quality or relevant data in the data flood.

6. Data Assumptions: it handles how the assumption is relevant to data processing.

7. Data comprehensiveness: which handles implications of uncovering data?

8. Data Scalability: It describing about to data scaling.

\section{Process challenges}

Data Capture, Data Arrangement, Data Transformation, data modeling and Data Visualization.

1. Data capture: which describes about how data captured from different sources.

2. Data Arrangement: which describes with how data brought into the line from various sources.

3. Data Transformation: which describes with when and how data should transform from original data to data suitable for analysis.

4. Data modelling: which describes what technique or Modelling or simulation used.

5. Data visualisation: which describes how data is visualized? Is that a normal person can understand the output? 
Management Challenges: Data Privacy, Security, Governance and ethical issues.

a) Data privacy: which describes how data privacy preserved? What Level of privacy?

b) Data Security: How secure is this enterprise data? How this data used?

c) Data ethics: what legal issues are in the data? What about ethical concern?

d) Data governance: how quality is the result? Any risk in applying any model?

\subsection{Big Data Challenges over Smart Healthcare System}

1. Understanding the knowledge of different patient sources like from Hospital, Clinic, office and home, etc.

2. Considerate unstructured clinical transcripts in the right context.

3. Effectively handling the huge volume of medical data and mining right information and biomarkers.

4. Provide right intervention to the right patient at the right time from huge volume of Personalised care for the patient

5. Hypothetically benefit all the components of a healthcare system, i.e., provider, payer, patient, and management.

6. Investigating genomic data is a computationally dangerous task and uniting with standard clinical data adds additional layers of complexity.

7. Health data are more personal thus patients expect extra privacy protection if they are going to participate in fully, Big Data applications in the healthcare space.

8. Taking the patient's behavioural data through various sensors; their different social interactions, and communications.

\subsection{Privacy Concerns in Big Data over Smart Healthcare System}

Privacy Concerns in big data over smart healthcare, domain privacy can be classified into two categories. Such as 1.Organization threats and 2.Systemic threats

Organization threats: Organizational threats would arise from the incorrect access of patient data by either private agents maltreating their privileges or external agents abusing liability of information systems, such as an employee who accesses data without any genuine need or an intruder that infiltrates organization's information infrastructure to snip data or extract it impractical.

Systemic threats: threats arise from an agent in the information flow chain abusing the revealed data elsewhere its anticipated use.

Information Security \& Interoperability: Few organizations store,health information in different registered formats. Using some of the Data formats can lead as a major hurdle in sharing patient's data among organizations as well as to medical and health policy research.

Data Security for Authentic Data Disclosure: In the healthcare sector, it is essential to share the data across the organizational limits to provision the further securities of multiple stakeholders, also, to supporting difficult with public health. Nevertheless, the release of patient's data might basis personally identify information as well sensitive information that may interrupt confidentiality as well cause socioeconomic consequences for the patient. Advances in technology have led to the consolidation of health records from multiple sources to a single research database which supports researchers engaged in the improvement of public health, clinical methods and health services in general.

\section{OPPORTUNITIES IN SMART HEALTHCARE SYSTEM}

Healthcare organizations have traditionally generated and utilized enormous data in size. Healthcare is well positioned to take benefit of contemporary tools that can extract more meaningful insights out of big data.

Big Data offers opportunities in Smart Healthcare System, i.e. who can use big data efficiently to improve the performance and reduce the cost.

Big data can contribute to lessen the cost of healthcare:

- Big Data further precisely isolate where education and preclusion are needed to produce healthier populations at lower costs

- Data takes the estimation out of health care. Despite using their judgment, physicians, and other providers can base protocols on scientific, likely evidence

Promoting Value and Innovation

- Big data offerings healthcare providers with the prospect to expand their value to patients with a more holistic, patient-centered approach to the delivery of care.

\section{SMART HEALTHCARE SYSTEM: OPERATIONAL STRUCTURE}

In this section we explore the basic operations performed in Smart Healthcare framework in our Smart Health Framework as shown in (Figure 3), we have been focused on some of the primitive Key Components in this context [10]

Sensing component: By using this Sensing element, it senses the user's health condition information continuously by placing sensing devices inside the patient's body. Moreover, this device collecting patient dynamic updated data and passes on to its dedicated servers which will help to query processing in realtime big data analytics.

Big Data Center: which yields in storing and processing the data collected from various sources?

Investigation Center: In this process doctors directly communicate with the patients and inquired, invoke the patient data in the investigation center depends on query, and analytic types and alarm will be generated, and message will be pass on to Investigation Centre

Healthcare Systems: in the Health Care System doctors decide based on the alarm type and suggest them to the appropriate health care system for better treatment they respond to the physician's request

Control Applications: this control application helps to consolidate the information with the clinicians' and masters' restorative information (from the healthcare system segment), the clinician in the perceptual focus may choose to send a few choices to the control/activation segment, which could include summoning a ready/update, illuminating a parental figure, or designing a smart device. At last, essential measures will be 
directed at the patients taking into account the conjured control applications.

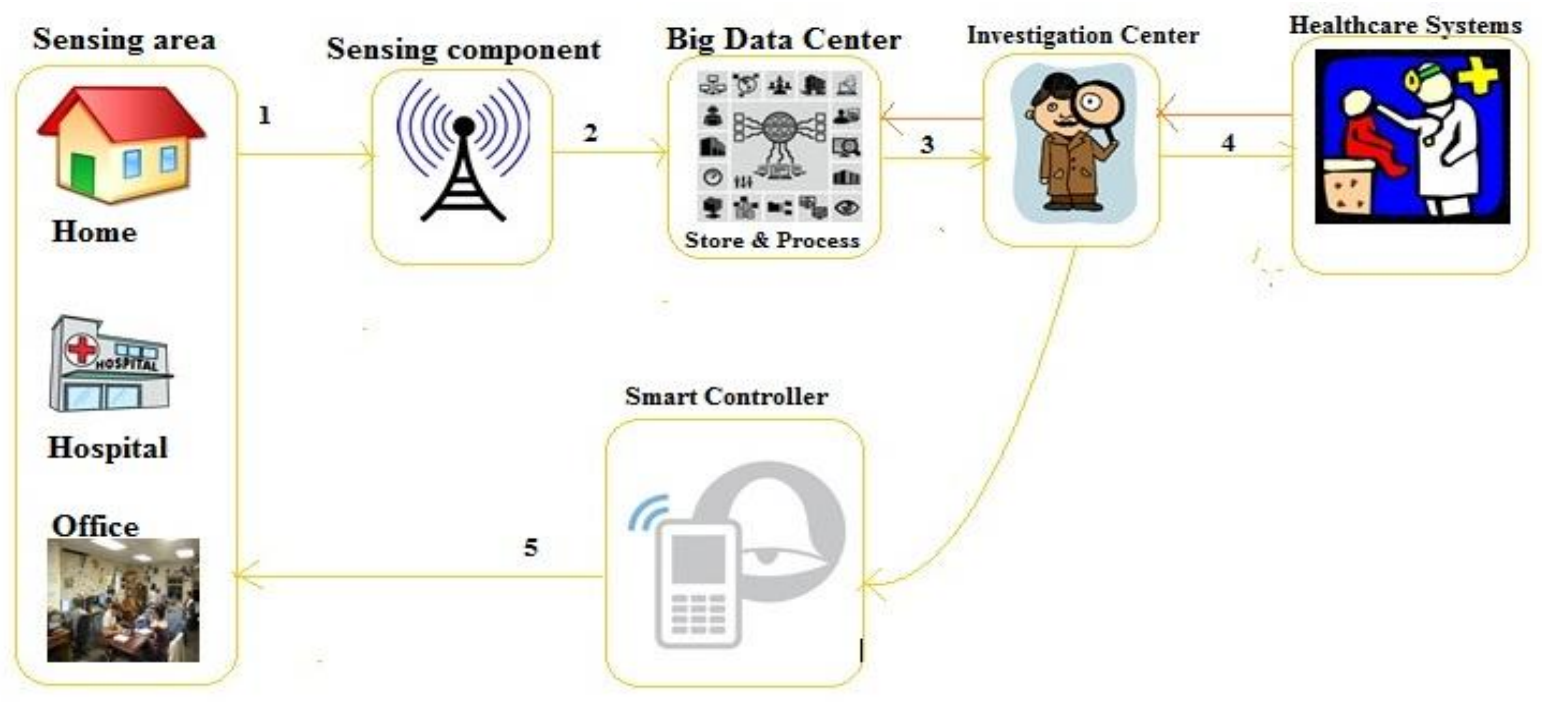

Figure 3.Smart Healthcare monitoring system

\section{BIG DATA ANALYTICS IN HEALTHCARE SYSTEM:}

Big Data analytics in health care or a combination of clinical advancement and technology overall. As the healthcare industry is constantly producing huge volumes of data in different practices, but it is practically impossible to manage such data over hard or soft copy formats. Data digitization can make easy to manage such a huge volume of data. Motivated by required requirements, the present generation favors "Data Analytics". This workable system supports a comprehensive assortment of health care, utilities to improve services and tackle problems in the healthcare sector as (Figure 4). The Big data platform is capable of processing terabytes and petabytes of data, as a result of which, data analysis becomes easier [3].

Big data analytics takes a novel approach to healthcare organisations to develop actionable discernments, organise their future vision, and increase the outcomes and reasonable time to value. This method is also useful to offer perceptive information to the healthcare enterprises regarding their management, planning, and the measurements. The assessed results can add to aid in enhancing the decision-making capacity of the top management.

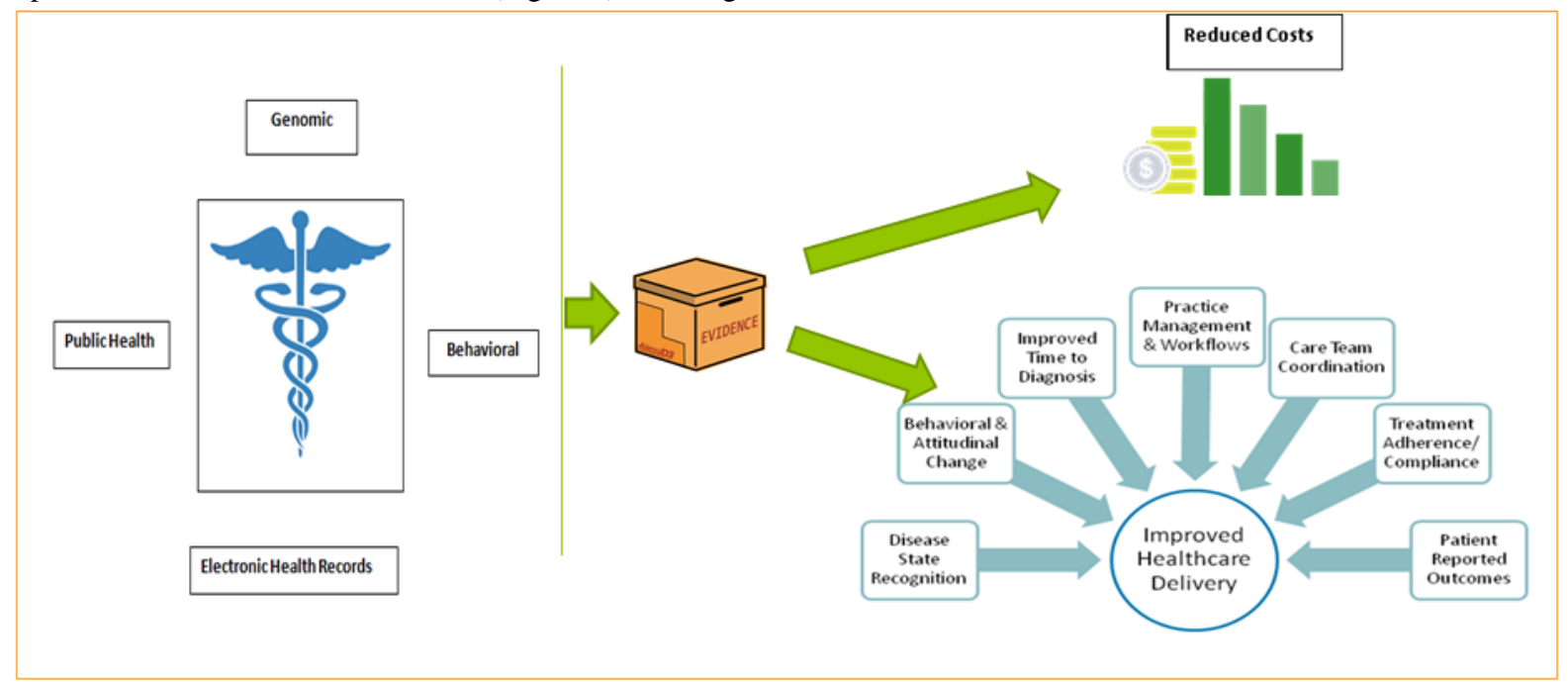

Figure 4. Big Data Analytics in Smart Healthcare System Data analytics in healthcare can be used to raise the standards in following fields

Public Health: by investigating disease patterns and recording disease outbreaks, public health issues can be value-added with an analytic approach. A high volume of data can aid to define needs, offer required services and predict and prevent the future crunches to benefit the population [5].

Electronic Medical Record or EMR: An EMR contains the standard (structured and unstructured) medical data that can assess with the data analytic approach to forecasting patients at risk and afford him with genuine care.

Patient Profile Analytics: Innovative analytics can be pragmatic to patients' profile for finding individuals who could benefit from the positive approach. It may embrace lifestyle deviations [4]. 
Genomic Analytics: The data analytic method can be effectively included in genomic analytics to make this approach a part of regular medical precaution decision process [6]

Fraud Analysis: This data analytics approach supports to examine some prerogative requests to decrease fraud cases. An effective analysis can help reduce fraud, waste, and abuse.

Safety Monitoring: Data analytics can also be used to analyze large real-time volumes of brisk data in hospitals. The approach may help in the safety supervisory and negative event prediction.

Evidence-based medicine: Combine and analyze a variety of structured and unstructured data-EMRs, financial and operational data, clinical data, and genomic data to match treatments with outcomes, predict patients at risk for disease or readmission and provide more efficient care[1].

Hadoop technology is successful in meeting the above challenges faced by the healthcare industry as MapReduce engine, and HDFS have the capability to process thousands of terabytes of data. Hadoop makes use of cheap commodity hardware, making it a pocket-friendly investment in the healthcare industry

\subsection{Uses}

- Isolate impending confined to little occurrences of illness and epidemics

- Develop analytical precision

- Create modified treatment plans

- $\quad$ Reduce infection proportions

- Afford cutting-edge supervisory provision

\subsection{Goals of Big Data Analytics in Healthcare System}

In Practice, the outline of the Smart Health framework is gone from supporting the way toward changing and enhancing the healthcare services conveyance model in different routes, for example[11]

- Take the promising position of the huge volume of information and give the right medication to the right patient at the apt time.

- There was a personalised consideration to the patient. Enhancing the personalization of the healthcare services prepare with the goal that people can screen and recognise their risk factor identification, precaution facilitation, and treatment, and empowers patients to live autonomously while being dealt with, that has an enormous constructive effect on their mental state, and then on their physiological state.

- Possibly profit all the constituents of a healthcare system, i.e., supplier, customer, patient, and management.

- Trusting on sensing-based screening and evaluation technology in the home and public locations to decrease the physical pressure on the location of hospitals and turn it into an electronic flow of information

- Changing the medication process from a reactive model to a proactive and precaution demonstrate that can altogether minimise the costs of hospital admissions for extraordinary occasions.

- Enabling better administration of clinical workloads and permitting the healthcare system to be viable organise the patients with the most astounding need.

- Supporting self-care indicative processes to screen vital signs and different estimations where this information imparted to a doctor, in individual or utilising teleconsultation, to play out to have a diagnosis. Moreover, diagnosis can at times be computerised for basic diseases, for example, flu.

\section{CONCLUSION}

In this paper challenges, opportunities and privacy with Big Data applications over "Smart Healthcare System" addressed. Big data have offered a new way to healthcare organisations to develop actionable insights, organise their future vision, boost up the outcomes and reduce time to value and also addressed SHS operational Structure (Smart Healthcare System).

\section{REFERENCES}

[1] Raghupathi W, Raghupathi V. Big data analytics in healthcare: promise and potential. Health Information Science and Systems. 2014;2:3. doi:10.1186/2047-2501-23

[2] IBM Global Business Services an Executive Report "Analytics: The real-world use of big data"

[3] Significant Benefits of Big Data Analytics In Healthcare Industry, 2016. Available from http://www.builtinla.com/blog/significant-benefits-bigdata-analytics-healthcare-industry

[4] IBM . IBM big data platform for healthcare." Solutions Brief. 2012.

[5] Manyika J, Chui M, Brown B, Buhin J, Dobbs R, Roxburgh C, Byers AH. Big Data: The Next Frontier for Innovation, Competition, and Productivity. USA: McKinsey Global Institute; 2011.

[6] IBM . Large Gene interaction Analytics at University at Buffalo, SUNY. 2012

[7] Smart use of Big data: https://www.healthcare.siemens.com/magazine/mso-bigdata-and-healthcare-2.html

[8] IDC iView "Extracting Value from Chaos", sponsored by EMC. 2011 June.

[9] IBM Global Business Services an Executive Report "Analytics: The real-world use of Big Data" 2013, pp. 116.

[10] Sakr S, Elgammal A, "Towards a Comprehensive Data Analytics Framework for Smart Healthcare Services", Big Data Res. 2016.

[11] How to improve healthcare systems with IoT and BigData,2016. http://www.kelltontech.com/kellton-techblog/how-improve-healthcare-systems-iot-and-big-data.

[12] Liang Y, Kelemen A (2016) Big Data Science and Its Applications in Health and Medical Research: Challenges and Opportunities. J Biom Biostat 7: 307. doi:10.4172/2155-6180.1000307 